\title{
Biometric Measurements: Average Intra-Ocular Lens Power for Sudanese Patients with Senile Cataract
}

\author{
Hiba Mohammed Elhassan Ali Elawad ${ }^{1}$, Mohammed Elhassan Ali Elawad ${ }^{2}$ \\ ${ }^{1}$ Faculty of Optometry and Visual Sciences, Department of Medical Photography, Alneelain University, Sudan, Khartoum \\ ${ }^{2}$ Faculty of Optometry and Visual Sciences, Department of Contact Lenses, Alneelain University, Sudan, Khartoum
}

Email address:

Hiba.m.elhassan@hotmail.com (H. M. E. A. Elawad)

\section{To cite this article:}

Hiba Mohammed Elhassan Ali Elawad, Mohammed Elhassan Ali Elawad. Biometric Measurements: Average Intra-Ocular Lens Power for Sudanese Patients with Senile Cataract. Optics. Vol. 4, No. 4, 2015, pp. 25-30. doi: 10.11648/j.optics.20150404.11

\begin{abstract}
The aim of the study is to determine the average intra-ocular lens power (IOL) for Sudanese patients with senile cataract. To contribute towards cataract treatment in Sudan and blindness prevention the goal of VISION 2020: The Right to Sight. Methods: Descriptive, cross-sectional, hospital based study was conducted in Makkah Eye Complex in Khartoum Alryad; in the period (October 2009-July 2010). Elderly patients (300); their age varied 60-95 years with senile cataract were asked to participate in the study. Participation was entirely voluntary. Information was collected by means of interview, clinical assessment and ocular measurements. The data were analyzed using SPAW Statistics-18 (2010) a modified version of SPSS. Results: The average horizontal corneal power (K1) for right eye (RE) 42.70D (SD \pm 1.625 ) and left eye (LE) 43.59D ( $\mathrm{SD} \pm 1.879)$. The average vertical corneal power $(\mathrm{K} 2)$ for $\mathrm{RE}$ 43.97D $(\mathrm{SD} \pm 1.710)$ and left $\mathrm{LE} 44.85 \mathrm{D}(\mathrm{SD} \pm 1.919)$. The average axial length is $23.72 \mathrm{~mm}$ ( $\mathrm{SD} \pm 1.143$ ) and $23.16 \mathrm{~mm}(\mathrm{SD} \pm 0.939)$ for the $\mathrm{RE}$ and LE respectively. The average IOL power for Extra-capsular cataract extraction (ECCE); with IOL to be implanted in the posterior chamber (PCIOL) was calculated to be 20.03D ( $\mathrm{SD} \pm 1.409$ ) for the $\mathrm{RE}$ and 20.19D ( $\mathrm{SD} \pm 2.639)$ for the LE. However, for Phacoemulsification (Phaco) the IOL power is calculated to be 20.06D ( $\mathrm{SD} \pm 1.740$ ) and 21.17D ( $\mathrm{SD} \pm 1.658)$ for the RE and LE respectively. Conclusions: The average corneal power was found be $(42-45 \mathrm{D})$, axial length $(23-24 \mathrm{~mm})$, where as the IOL power (20-21D); similar to international standards. In this study the biometric measurements were considered pre-operatively for all patients studied. Changes in biometric measurements after cataract extraction were not investigated since it wasn't part of the research objective; however, further studies are recommended to determine the influence of cataract extraction in corneal power, curvature, thickness, depth of anterior chamber and axial length.
\end{abstract}

Keywords: Senile Cataract, Biometric Measurements, Corneal Power, Axial Length, Inta-Ocular Lens, Ophthalmic Ultrasonography, Phacoemulsification, Extra-Capsular Cataract Extraction

\section{Introduction}

According to WHO worldwide estimation every 5 seconds an adult person becomes blind. Ninety percent of blind people live in low income countries. Eighty percent of the causes are preventable or treatable. Cataract is the leading cause of blindness, accounting for $39 \%$ of blindness worldwide [1].

The most common eye disorder, affecting older people is Senile-Cataract Surgical removal of cataract remains the only proven therapy and can be successful in restoring vision [2-5].

Cataract surgery has been shown by multiple studies to be one of the most cost-effective health interventions, and leads to a dramatic increase in quality of life and productivity for many patients $[6,7,8]$.

An intraocular lens is the ideal optical correction and is preferable if there are no contraindications (although visual acuity may be equal in spectacles, contact lenses and intraocular implant, the retinal image is enlarged considerably in spectacle corrections 20 to 30 percent, moderately in contact lens correction 6 to 7 percent, and minimally in an intraocular lens about 1 percent $[4,9,10]$.

Suitable power for the IOL is calculated by using a special formula which incorporates keratometry readings and length of the globe as determined by A-scan ultrasonography $[4,10]$.

Prevalence of blindness in Sudan is estimated to be $1 \%$. Cataract is the leading cause of blindness, accounting for $55 \%$ of blindness [6-8, 11, 12-16]. The estimated economic loss in Sudan due to blindness is 1.5 million $\$$ per a day; 550 million 
$\$$ annually. Estimated annual cost for prevention of blindness is 1 million $\$,[15,16]$.

In Sudan cataract output and surgical rate (CSR) has increased steadily from 2003 to 2009. In 2009, cataract output was 72024 (range: 55- 37305). CSR was 2025 (range: 76-7073). More than $98 \%$ of surgeries with IOL implant in 2009 compared to less than $20 \%$ in 2002 [11, 12-16]. Nevertheless; cataract remains to be the leading cause of blindness in Sudan. There is, however, little research data to support and contribute towards its treatment.

\section{Materials and Methods}

The study was descriptive - cross sectional; hospital based. However, the study population consisted of elderly patients with senile cataract attending ophthalmic ultrasonography clinic: A-B scan department attending primarily for IOL measurement and posterior segment evaluation. The research was conducted using standard instruments and protocol fig. 1.

\subsection{Research Protocol}
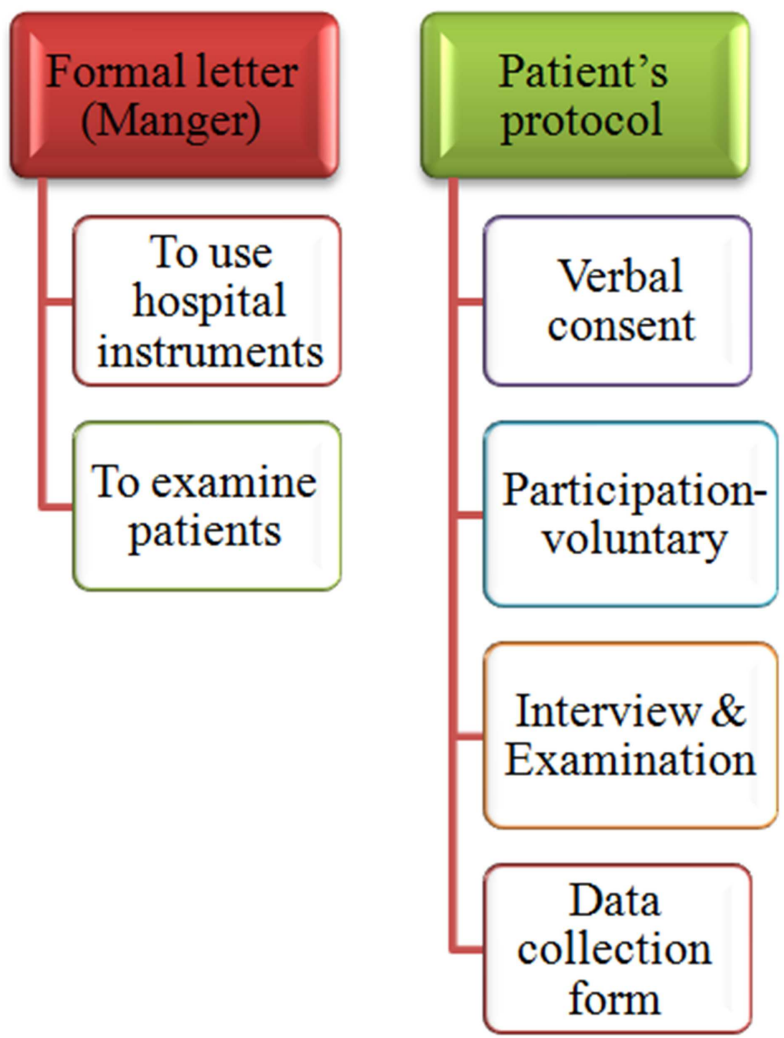

Fig. 1. Research protocol.

\subsection{Patient's Selection Criteria}

- Patient age 60 years or more.

- Patients with ocular manifestation of senile cataract; with some degree of lenticular opacity ranging from mild to severe; however, they ready to undertake cataract surgery in one or both eyes.

- Absence of other ocular diseases was mandatory.

- Subjects with history of systemic disease were included.
Presence of systemic diseases was allowed, but without ocular manifestations.

- Both genders are considered.

- Subjects attending from other states were included in the study.

Patient's exclusion criteria:

- Foreigners.

- Ocular co-morbidity: Majority of the elderly patients have multiple ocular pathologies.

\subsection{Research Instruments and Clinical Procedures}

The instruments used include the following:

- Slit-lamp bimicroscope: CSO-Italy model SL 990-5 (examination of anterior ocular segment and ocular media) see fig. 2.

- Nidek KM-500 hand held auto-keratometer (measurement of corneal curvature and power).

- HEINE BETA 200 Direct ophthalmoscope (evaluation of posterior ocular segment for patients with observable retinal reflex).

- Alcon: Ultrascan imaging system and Nidek US-4000 Echo-scan (A-mode and B-mode).

Achieving the targeted postoperative refraction requires measuring axial length (A-mode) accurately, determining corneal power (Keratometric measurements), and using the most appropriate IOL power formula (PCIOL or Phaco).

Corneal powers: In this study automated keratometry were obtained using Nidek KM-500 hand held keratometer. The KM-500's was used because it has unique viewing and alignment window, light weight, allows comfortable binocular observation, simple operation and measurement (see fig. 3: a, b).

The clinical procedure used to determine the refractive power of the cornea included: Viewing the eye through the window and moving the KM-500 towards the patient's eye. When the mire ring appeared on the cornea the unit slowly moved closer. Target ring start blinking automatically for fine alignment. The KM-500 fired automatically when properly focused. Readings appeared instantly on the LCD display (reading were over with a beep). Both left and right readings were displayed simultaneously.

The A-mode: Is a uni-dimensional display because only one axis is displayed at a time. The ocular biometric measurements were obtained using the A-mode. However this study was only concerned with axial length of the eye and the lens power. Alcon: Ultra-scan imaging system (see fig. 4: a, b, c) and Nidek US-4000 Echo-scan (fig. 5: a, b) were both used to display the A-mode. Although they both shared the same clinical procedure they differ: the Alcon required entering the keratomteric measurement to calculate the correct power for the IOL, while Nidek provided data automatically, and the later could also be used to obtain pachometry. Both instruments were useful to assess the posterior segments health (B-Mode).

The patient was seated comfortably fixating at a light source placed $6 \mathrm{~m}$ away, One drop of local anesthesia were instilled into patients eye, hand-held probe (sterilized using 
normal saline) was placed on the cornea (applied centrally), when alignment was achieved the system automatically display the A-mode ultra-sonogram with accurate axial length (A mean was calculated from 10 repeated measurements made of each axial length) and IOL power. To calculate the IOL power: A- constant $114.5 / 118$ and 118.5 were used for ECCE+PCIOL and phaco's respectively. The right eye (RE) measurements were obtained first followed by the left eye (LE).

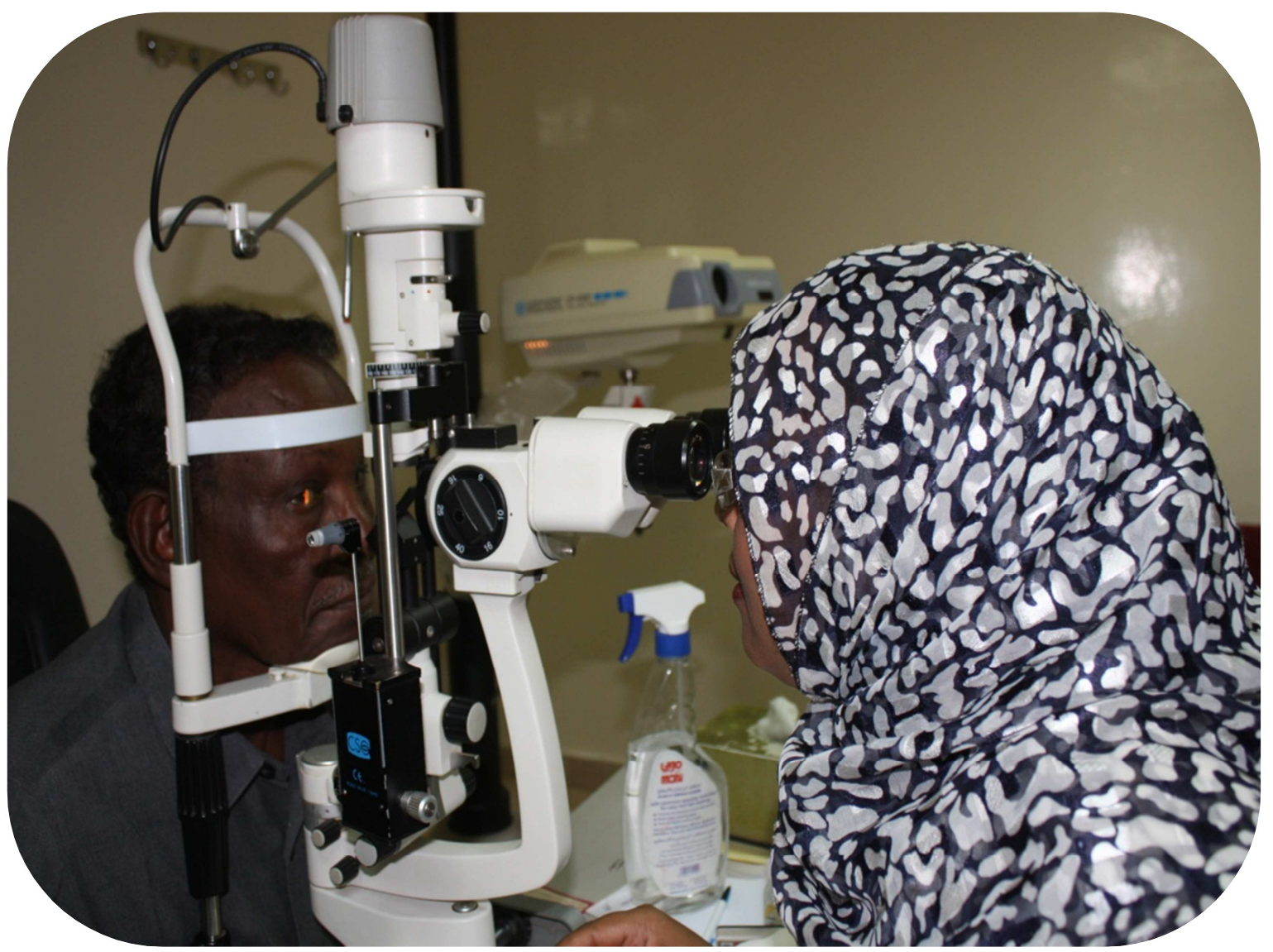

Fig. 2. Slit-lamp bimicroscope: examination of anterior ocular segment and ocular media.

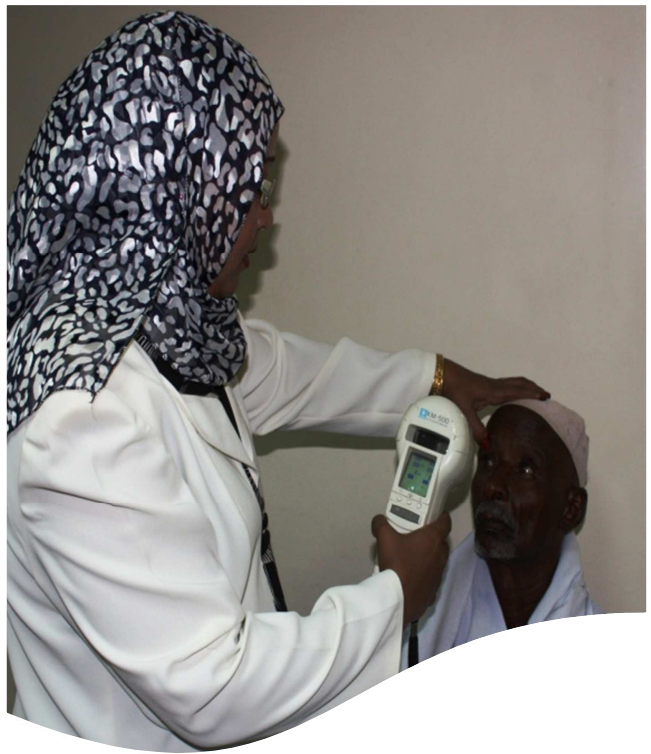

(a)

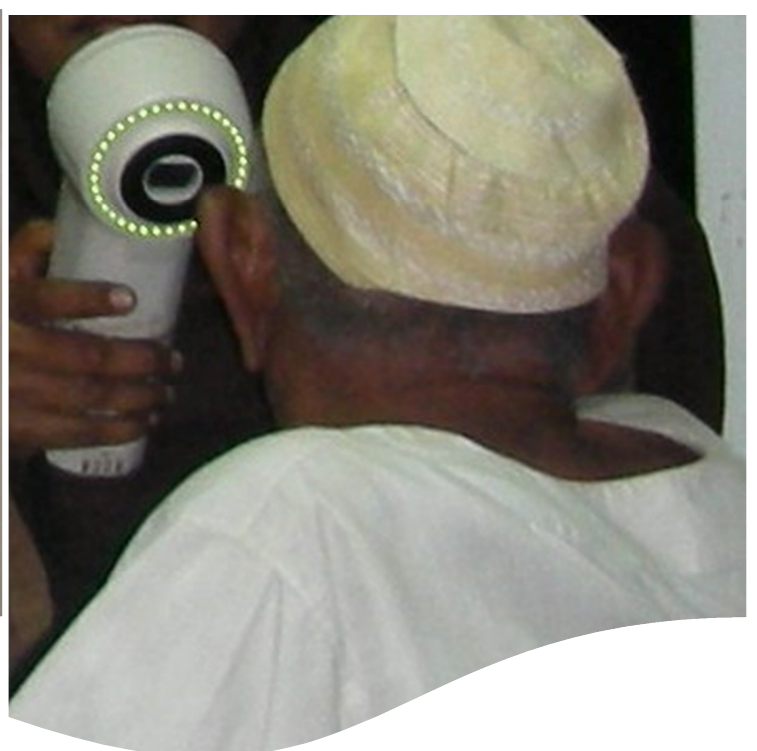

(b)

Fig. $3(\boldsymbol{a}, \boldsymbol{b})$. Nidek KM-500 hand held keratometer in use. 


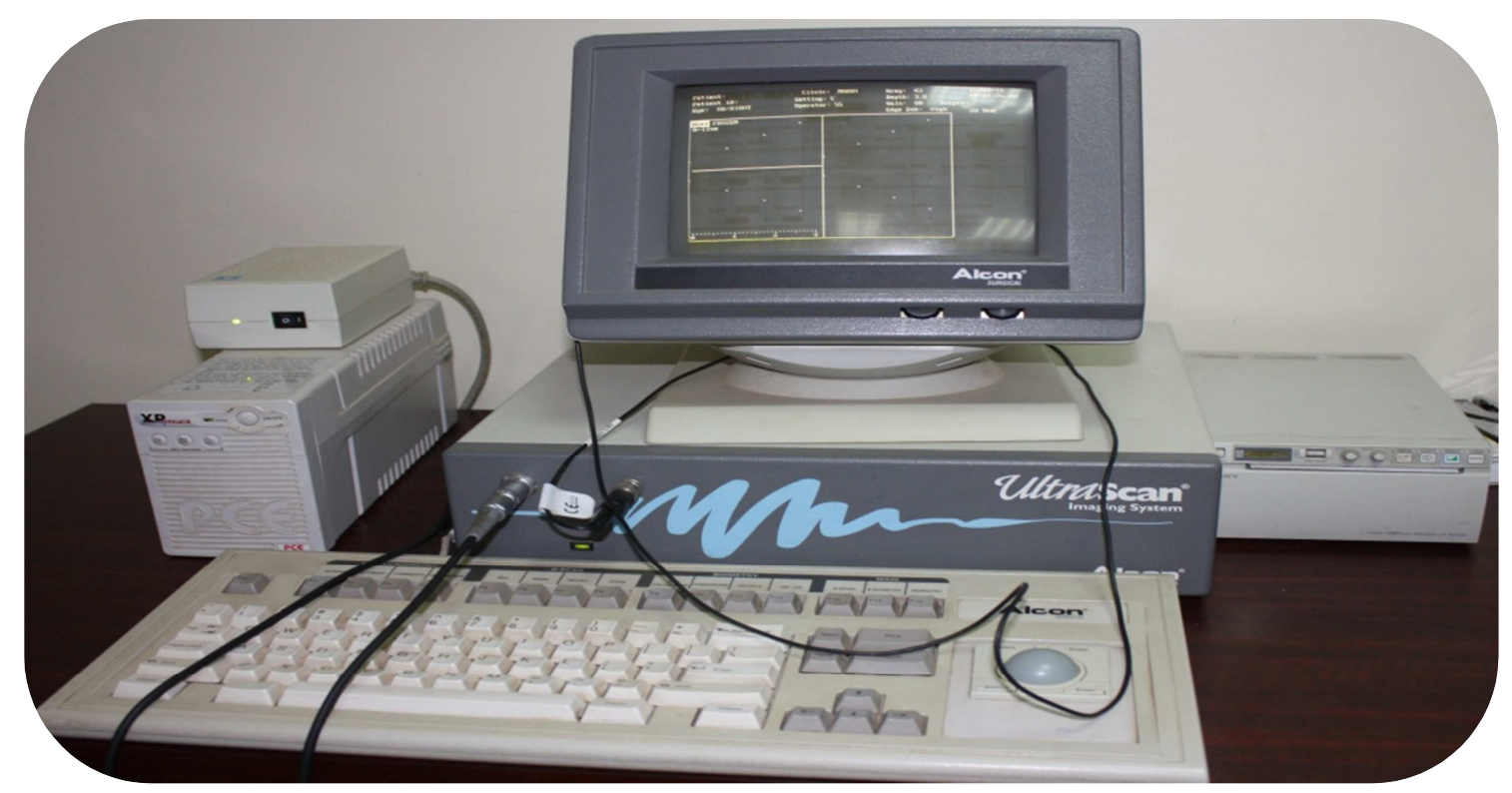

(a)

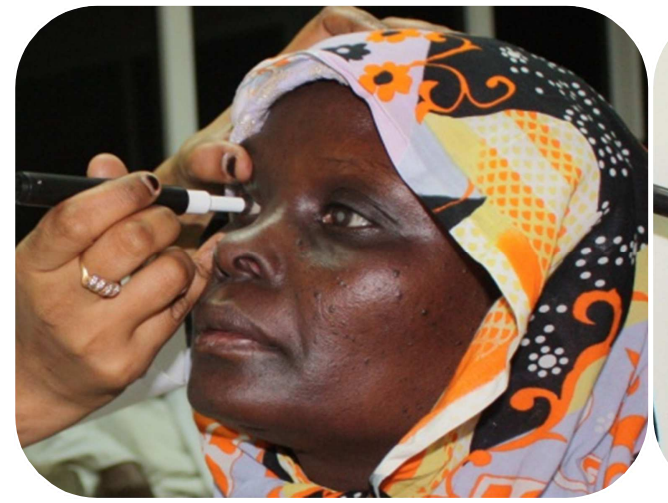

(b)

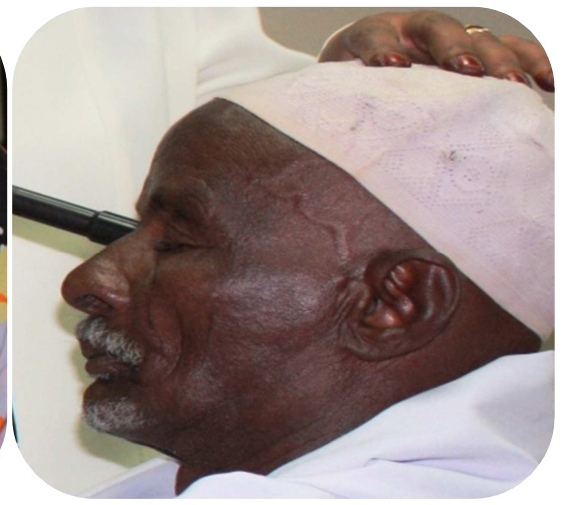

(c)

Fig. $4(\boldsymbol{a}, \boldsymbol{b}, \boldsymbol{c})$. Alcon; Ultrascan imaging system used for A-mode and B-mode.
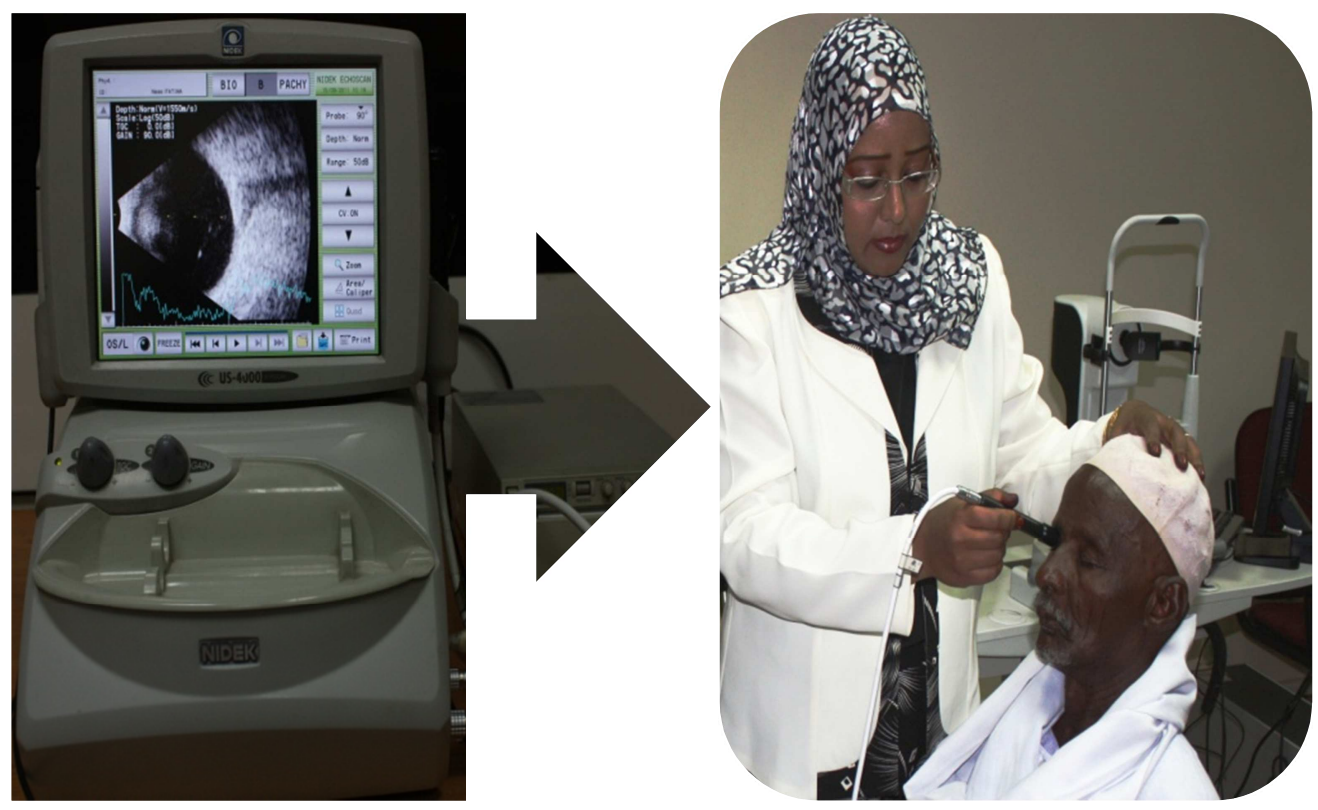

Fig. 5 (a, b). B-scan. Posterior segment evaluation; Nidek US-4000 EchoScan. 


\section{Results}

Table 1. Descriptive statistics of corneal powers (D).

\begin{tabular}{|c|c|c|c|c|c|c|c|c|}
\hline Statistics & & Mean & SE & Median & Mode & SD & Min & Max \\
\hline Horiz. & $\mathrm{RE}$ & 42.70 & 0.217 & 42.50 & 41.50 & \pm 1.625 & 39.00 & 47.25 \\
\hline K1 & LE & 43.59 & 0.290 & 42.63 & 43.75 & \pm 1.879 & 38.25 & 50.25 \\
\hline Vertic. & $\mathrm{RE}$ & 43.97 & 0.229 & 43.63 & 43.25 & \pm 1.710 & 40.75 & 48.75 \\
\hline K2 & LE & 44.85 & 0.296 & 44.75 & 45.00 & \pm 1.919 & 41.75 & 51.75 \\
\hline
\end{tabular}

Table 2. Descriptive statistics of axial length ( $\mathrm{mm}$ ).

\begin{tabular}{|c|c|c|c|c|c|c|c|c|}
\hline Statistics & & Mean & SE & Median & Mode & SD & Min & Max \\
\hline $\mathrm{H}$ & $\mathrm{RE}$ & 23.72 & 0.154 & 23.67 & 23.3 & \pm 1.143 & 21.63 & 29.75 \\
\hline AL & LE & 23.16 & 0.148 & 23.15 & 22.73 & \pm 0.939 & 20.26 & 24.87 \\
\hline
\end{tabular}

Table 3. Descriptive statistics of IOLs power (D).

\begin{tabular}{|c|c|c|c|c|c|c|c|c|}
\hline Statistics & & Mean & SE & Median & Mode & SD & Min & Max \\
\hline ECCE+ & $\mathrm{RE}$ & 20.03 & 0.222 & 19.80 & 19.50 & \pm 1.409 & 17.00 & 24.00 \\
\hline PCIOL & $\mathrm{LE}$ & 20.19 & 0.466 & 20.00 & 20.00 & \pm 2.639 & 15.50 & 30.50 \\
\hline \multirow{2}{*}{ Phaco } & $\mathrm{RE}$ & 20.06 & 0.435 & 20.50 & 19.00 & \pm 1.740 & 16.00 & 22.00 \\
\hline & $\mathrm{LE}$ & 21.17 & 0.552 & 21.50 & 21.50 & \pm 1.658 & 18.50 & 23.50 \\
\hline
\end{tabular}

ECCE, Extra - capsular cataract extraction; PCIOL, IOL to be implanted in the posterior chamber; Phaco, Phacoemulsification.

\section{Discussion}

Corneal powers: Table (1) results indicate that the vertical meridian of the cornea is steeper than the horizontal one; resulting in an increase in against-the-rule astigmatism in older people. Most cross-section studies of refractive error and age confirm this trend $[9,10]$.

The average axial length is $23.72 \mathrm{~mm}(\mathrm{SD} \pm 1.143)$ and $23.16 \mathrm{~mm}(\mathrm{SD} \pm 0.939)$ for the RE and LE respectively (see table 2). International standard agree with these findings which indicate that the average axial length $23-24 \mathrm{~mm}$. Nevertheless, it had been observed to be shorter in hypermetropes and longer in myopes.

Table (3) showed that the average IOL power for Extracapsular cataract extraction (ECCE); with IOL to be implanted in the posterior chamber (PCIOL) was calculated to be 20.03D $(\mathrm{SD} \pm 1.409)$ for the $\mathrm{RE}$ and 20.19D $(\mathrm{SD} \pm 2.639)$ for the LE. However, for Phacoemulsification (Phaco) the IOL power was calculated to be $20.06 \mathrm{D}(\mathrm{SD} \pm 1.740)$ and 21.17D ( $\mathrm{SD} \pm 1.658$ ) for the $\mathrm{RE}$ and $\mathrm{LE}$ respectively.

Descriptive statistics of IOLs power revealed the average ECCE+PCIOL power required to substitute the crystalline lens was $20 \mathrm{D}$ and for phaco $20-21 \mathrm{D}$, Thus the phaco power is slightly greater than the ECCE+PCIOL.

\section{Conclusions and Recommendations}

The average corneal power is found be (42-45D), axial length (23-24mm), where as the IOL power (20-21D).

It has been noticed that pre-operative measurements for IOL calculation in the hospital was usually obtained for one eye (eye requiring operation); binocular measurements are recommended to avoid the possible binocular vision problems. Thus, the refractive power of the eye can deliberately be altered either toward emmetropia or iseikonia, depending on estimated future status of the opposite eye.

Limitation of the study: comparative studies with patients originating from the same racial or ethnic background living in similar cultures and conditions are required.

Recommendations: Clinical use and implications of standard IOLs, aspheric IOLs, toric, monovision and presbyopia-correcting IOL implants deserve clinical investigations.

\section{Acknowledgements}

Authors express gratitude to the manger of Makkah Eye Complex Dr. Lakho, K., for giving us the opportunity to use hospital clinics and instruments; and the elderly patients for scarifying their time to participate in our study.

\section{References}

[1] Blindness Prevention Program: World Health Organization, WHO Report htpp//www.Sudanembassy.org/contemporaryLooks/blindness htm.

[2] Michaels, David D., 1993. Ocular Disease in the Elderly. In Rosenbloom, Alfred A., Morgan Meredith W., 1993. Vision and Aging, 2nd ed. Butterworth-Heineman. Chapter 4. pp. 116-147.

[3] Morgan, Meredith W., 1993. Normal Age-Related Vision Changes. In Rosenbloom, Alfred A., Morgan, Meredith W., 1993. Vision and Aging, 2nd ed. Butterworth-Heinemann. Chapter 6: 178-195.

[4] Kanski, Jack J., 1994. Clinical Ophthalmology, 3rd ed. BH publication. Chapter 9: pp. 286-306.

[5] HAI. 2000. Common Eye Disorders in Older People. AGE WAYS 53: 12. 
[6] Elawad M., and Elawad, H., 2011(a). EyeZone: Vision related quality of life in Sudanese patients with senile cataract (preoperative cases). EyeZone Issue 35: January/Feburary 2011. Studies \& research: pp 34-39.

[7] Elawad, H., and Elawad, M., 2011 (b). The Self-Expressed Needs for Sudanese Patients with Senile Cataract. In Journal of Clinical and Experimental 2011, 2:5 April 2011. Avaliable online at: OMICS Publishing group (www.omicsonline.org). http://dx.doi.org/10.4172/2155-9570.1000158.

[8] Elawad, H., and Elawad, M., 2011 (c).Age-related cataract: Senile cataract in Sudan. AL-Basar International Foundation 2011.

[9] Bennett, Edward S., Remba, Melvin. J. and Weissman, Barry A., 1993. Contact Lenses and The Elderly Patient. In Rosenbloom Alfred A., and Morgan Meredith W., 1993. Vision and Aging, 2nd Edn. Butterworth-Heinemann. Chapter 9: 252-256.

[10] Goss, David A., and Wess. Roger W., 2002. Introduction to The Optics of the Eye. Butterworth-Heinemann. Chapter 8: 186, Chapter 9: 204.
[11] Etya'ale, D., 2002. Cataract in Sudan, Current Situation, Projections and Proposed Outlines for Control. Paper represented in VISION 2020: The Right to Sight Workshop (11-16 Feb, 2002).

[12] NPPB. 2009. Report: Number of Cataract Surgeries Done in 2008 in Sudan. National Program for Prevention of Blindness.

[13] NPPB. 2010. Report: Number of Cataract Surgeries Done in 2009 in Sudan. National Program for Prevention of Blindness.

[14] Binnawi, Kamal H., Alshafae, B. and Hassan, A., 2011 Cataract Surgical Rate in Sudan. Paper presented in Europian Society for Cataract and Refractive Surgery (ESCRS), Feburary, 2011.

[15] Binnawi, Kamal H., Alkhair, B. and Hassan, A., 2011. Cataract surgery in Sudan: Output, rate, coverage, quality and outcome. Paper represented in Sudanese Ophthalmological Society: The 17th Scientific Conference 13-14 April 2011.

[16] Binnawi, Kamal H., 2011. Less than 10 years for 2020: Overview of progress in preventing blindness in Sudan. Paper represented in Sudanese Ophthalmological Society: The 17th Scientific Conference 13-14 April 2011. 